\title{
THE MUSICOS PROJECT: MULTI-SITE CONTINUOUS SPECTROSCOPY
}

\author{
CLAUDE CATALA, JACQUES BAUDRAND, TORSTEN BÖHM \\ Observatoire de Paris, Section de Meudon, 92195 Meudon Principal Cedex, \\ France
}

\author{
BERNARD H. FOING \\ Institut d'Astrophysique Spatiale, BP 10, 91371 Verrières-le-Buisson Cedex, \\ France
}

\section{INTRODUCTION}

Many scientific programs, most of them linked to stellar physics (such as asteroseismology, stellar rotational modulation, surface structures, Doppler imaging, Zeeman-Doppler imaging, variable stellar winds) require a continuous spectroscopic coverage during several days.

MUSICOS (for MUlti-SIte COntinuous Spectroscopy) is an international project for setting up a network of high resolution spectrometers coupled to telescopes of the $2 \mathrm{~m}$ class, well distributed around the world, and partly dedicated to continuous spectroscopy.

The strategy to reach this objective was defined during two workshops organized at Paris-Meudon Observatory in 1988 and 1990, and consists of three steps: 1) organize multi-site spectroscopic campaigns using resident instruments on various telescopes around the world and transportable fiber-fed spectrographs where adequate spectroscopic equipment is not available; 2) design and develop a cross-dispersed echelle spectrograph, well suited for the scientific programs that require multi-site observations; 3 ) propose this MUSICOS spectrograph for duplication at several collaborating sites.

\section{THE MUSICOS SPECTROGRAPH}

We have now completed phase 2 of this strategy. We have constructed the MUSICOS spectrograph, which is now ready, and installed at the "Bernard Lyot" $2 \mathrm{~m}$ telescope at $\mathrm{Pic}$ du Midi. This spectrograph has been designed to meet the requirements of scientific programs that need multi-site observations. Figure I shows the optical layout of the spectrograph. It is a cross-dispersed spectrograph, covering most of the visible domain in 2 exposures (380-540 $\mathrm{nm}$ and $540-870 \mathrm{~nm}$ ), with a resolving power of 38000 . The spectrograph is fed by 2 fibers, giving the possibility to record simultaneously 2 spectra with interleaved orders. Other characteristics of this spectrograph include high stability and easy transportability. The cost of the spectrograph was kept low $(<100,000$ USD, including detector). Finally, we developed a dedicated automatic reduction software for the MUSICOS spectrograph. Table I indicates the performances of this instrument, as obtained at Pic du Midi 2m "Bernard Lyot" telescope, for an A0 


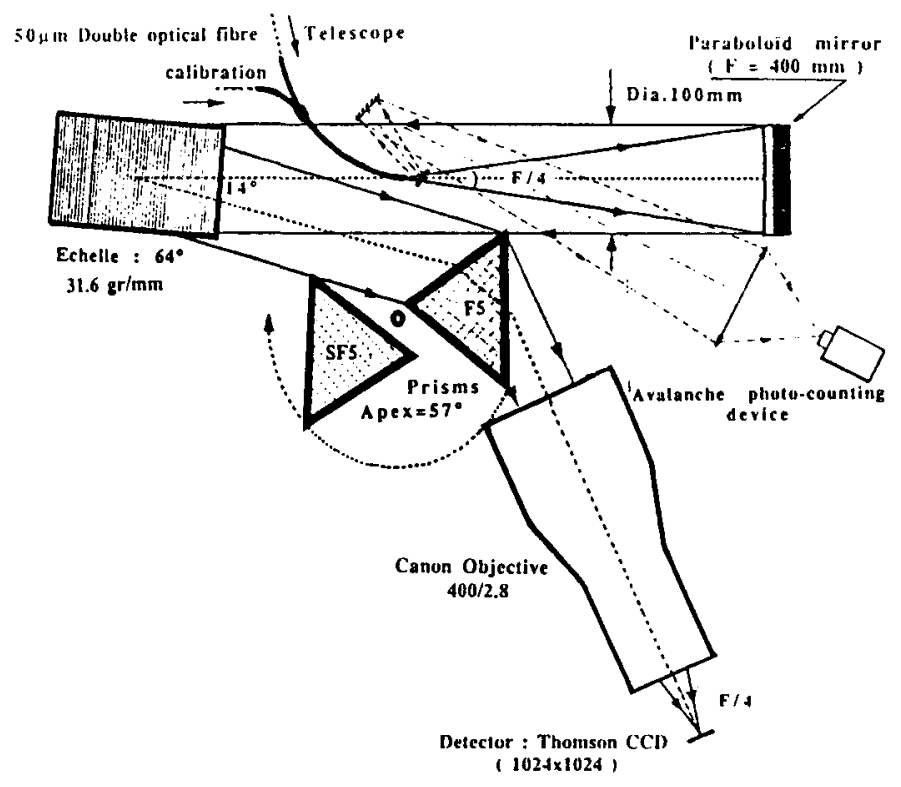

FIGURE I Optical layout of the MUSICOS spectrograph

star with $\mathrm{m}_{\mathrm{V}}=7$, with a thick coated CCD, in one hour.

\begin{tabular}{ccc}
\multicolumn{3}{c}{ TABLE I } \\
\hline$\lambda(\mathrm{nm})$ & S/N & CCD \\
& & Q.E. \\
\hline 400 & 125 & 0.12 \\
500 & 320 & 0.30 \\
600 & 405 & 0.45 \\
700 & 380 & 0.40 \\
800 & 300 & 0.30 \\
\hline \hline
\end{tabular}

The instrument, its dedicated reduction software, as well as its performances on the sky are fully described in Baudrand and Böhm (1992).

\section{THE MUSICOS 1989 CAMPAIGN}

In December 1989, we organized a vast multi-site spectroscopic campaign, with the philosophy presented above. Three scientific programs were followed: 1) search for nonradial pulsations in the Be star 48 Per; 2) search for co-rotating structures in the wind of the Herbig Ae star AB Aur; 3) Doppler Imaging and flare patrol for the RS CVn system HR 1099.

The campaign was organized around a core program, involving telescopes in Hawaii, Kitt Peak, ESO La Silla, France OHP, Crimea, and China. Two spectrographs had to be transported to remote sites for this campaign: the fiberfed ISIS spectrograph was transported from Meudon to the $2.16 \mathrm{~m}$ telescope in Xinglong, while the ISIS-bis spectrograph travelled to Hawaii, to be mounted on the UH $2.2 \mathrm{~m}$ telescope. 

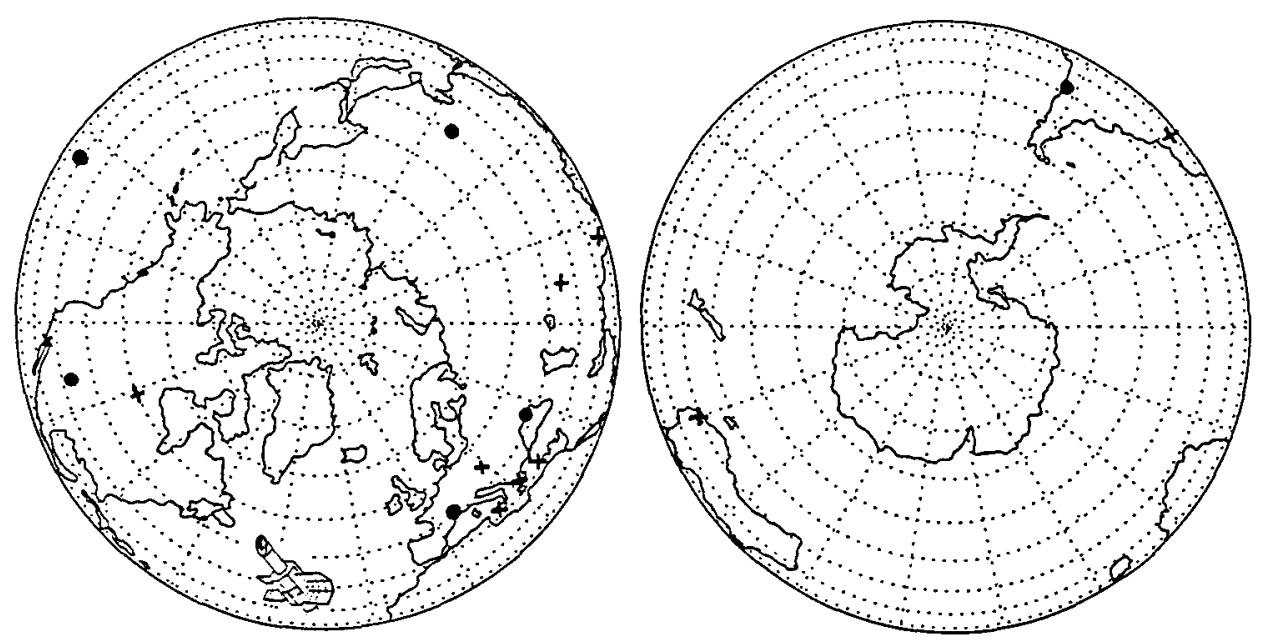

FIGURE II Distribution of the sites involved in the MUSICOS 1989 campaign. The filled symbols correspond to sites of the core program, while "pluses" indicate additional sites.

In addition to this core program, a large number of astronomers all over the world decided to contribute to this campaign, after it was announced in an IAU circular. Altogether, up to 17 telescopes were pointed toward the targets, including IUE. Figure II shows the distribution of the sites on the Earth.

The MUSICOS 1989 campaign was a great success, with some major scientific impact. The full organization of the campaign, as well as some preliminary results can be found in Catala and Foing (1990). The final scientific results will be published shortly (Foing et al., 1992).

We are currently planning a new campaign for December 1992, involving the newly developed MUSICOS spectrograph, that we intend to transport to the $2.2 \mathrm{~m}$ telescope in Hawaii. Our two other fiber-fed spectrographs, ISIS and ISIS-bis will be transported to India and China.

\section{REFERENCES}

Baudrand, J., Böhm, T. 1992, $A \& A$, in press

Catala, C., Foing, B.H. 1990, in Proceedings of the second MUSICOS workshop, Meudon, C. Catala and B.H. Foing (eds.), Observatoire de Paris press

Foing, B.H., Char, S., Jankov, S., Catala, C., Ayres, T., Neff, J.E., Simon, T., Zhai, D.S., Cutispoto, G., Catalano, S., Rodono, M., Akan, C., Baudrand, J., Cao, H., Cuby, J.G., Czarny, J., Dreux, M., Felenbok, P., Ghosh, K., Guérin, J., Huang, L., Hubert, A.M., Jiang, S., Jinxin, H., Keskin, V., Mavridis, L., Nolthenius, R., Zihe, G. 1992, $A \mathcal{B} A$, submitted 